\title{
Basic accountability to stop ill-treatment (BASI): study protocol for a cluster randomized controlled trial in rural tanzania
}

\author{
Redempta Mbatia*, Jessica Cohen, Martin Zuakulu, Appolinary Bukuku, Shikha Chandarana, Eliudi Eliakimu, Sisty Moshi and Elysia \\ Larson
}

Tanzania Health Promotion Support, Tanzania

\begin{abstract}
Background: Poor health system experiences negatively affect the lives of poor people throughout the world. In East Africa there is a growing body of evidence of poor quality that in some cases goes as far as disrespectful or abusive care. This study will assess whether community feedback through report cards (with and without non-financial rewards) can improve patient experience. The patient experience includes aspects of patient dignity, autonomy, confidentiality, communication, timely attention, quality of basic amenities, and social support.

Methods/Design: This cluster-randomized controlled study will randomize 75 primary health care facilities in rural Pwani Region, Tanzania to one of three arms: private feedback (intervention), social recognition reward (intervention), or no feedback (control). Within both intervention arms, we will give the providers at the study facilities feedback using the baseline data. Facilities randomized to the social recognition reward arm will also have a star-chart displaying their achieved level of patient experience publicly posted and potential recognition from senior officials at the local NGO and/or the Ministry of Health. We will use surveys with parents/ guardians of sick children to measure patient experience, and surveys with healthcare providers to assess potential mechanisms of effect.
\end{abstract}

Conclusion: Results from this study will provide evidence for whether, and through what mechanisms, patient reported feedback can affect interpersonal quality of care.

\section{Background}

There is growing evidence that patients experience low quality healthcare in low- and middle-income countries [1-3]. Providers often lack the knowledge, training and incentives to examine, diagnose, and treat patients effectively. A survey of Tanzanian rural public facilities in 2014 found that providers had low compliance (24.1\%) to clinical guidelines for handling maternal and neonatal complications and diagnostic accuracy was only $43.9 \%$ [4]. Poor quality also extends to interpersonal care: across seven sub-Saharan African countries only $39 \%$ of parents/guardians were told about danger signs that they should look for in their sick child [5]. A particularly egregious case of poor patient experience is disrespect and abuse: disrespectful treatment during labor and delivery ranges from $12 \%-28 \%$ of deliveries in East Africa and in one region of rural Tanzania, 14\% of women reported disrespectful care during their most recent outpatient care visit for their child [Larson et al, In Press] [6-8].

Poor patient experience is a marker of a poorly functioning health system. There are several consequences, two of which are discussed here. First, disrespectful care violates individuals' fundamental rights to be treated with dignity. Second, data from both low- and highincome countries suggest that interpersonal quality of health care is a primary determinant of patient utilization of future care $[9,10]$. Experiences and expectations of poor treatment can cause patients and caregivers to avoid or delay seeking care. Poor communication from providers can influence patient adherence to prescribed treatments and to recommended preventive activities [11-13].

Three field experiments in sub-Saharan Africa have found large behavioral improvements with non-financial interventions. In the first, providing feedback reports on health worker performance led to significant improvements in healthcare delivery and health outcomes $[14,15]$. In the second, social recognition through letters from employers and public interviews, led to improved test scores by health workers [16]. In the third, social recognitions through visible charts displaying the public health agents' performance led to increased condom sales [17]. Our current study builds on these findings in a new context, assessing whether community feedback through feedback reports, with and without social recognition, can improve patient experience. While providing feedback from patients has been shown to affect provider technical behavior in some contexts, patient feedback has not been extensively explored for patient experience [14,18-22].

In this study, we aim to address three causes of poor patient experience: normalization of poor quality, low attention to poor quality, and lack of accountability to the community. Private reports of data on patients' experience serve to increase attention to the problem by giving providers specific information on areas where patients feel they need improvement. By including benchmarks for achievement and discussions with leaders from local organizations, the feedback aims to further decrease normalization of the problem. Social recognition by way of public reporting of providers' success in delivering high quality care, incentivize providers to improve and hold them accountable

${ }^{\star}$ Correspondence to: Redempta J Mbatia, Executive Director, Tanzania Health Promotion Support 3rd Floor, Coco Plaza Bld, Plot 254 Masaki, Tanzania, Tel: +255-22-2923107; E-mail rmbatia@thps.or.tz

Received: March 27, 2018; Accepted: April 18, 2018; Published: April 21, 2018 
to the communities they serve. This study will provide evidence for which mechanisms affect patient experience and how communities can be involved to improve quality. The ultimate impact of the study is increased respect and communication during visits for sick children, which is both intrinsically valuable and has been associated with improved health outcomes.

\section{Methods/design}

\section{Study area}

The study is being conducted in four rural districts of Pwani Region, Tanzania (Bagamoyo, Kibaha Rural, Kisarawe, and Mkuranga). Sixty-seven percent of the population in the region resides in rural communities, where health services are primarily delivered through dispensaries and health centers. Estimates for literacy rate in the region were as low as 50\% in 2002 [23]. Data from the Demographic and Health Survey indicate that this area is one of the poorer areas in Tanzania, with $53.7 \%$ of the population in the bottom two wealth quintiles, and only $1 \%$ of the rural Pwani population in the highest wealth quintile [24].

Tanzania Health Promotion Support (THPS), a local nongovernmental organization supporting this study, currently supports government-managed health facilities in the study districts. Study facilities are government-managed, THPS-supported, primary care clinics (dispensaries) that had at least 450 sick child visits during the period from October 2016-June 2017. In one district we included two facilities with fewer than 450 visits in order to have a minimum of 15 facilities per district. We selected primary care clinics (known as dispensaries in Tanzania) for the study, because they are the lowest level of the Tanzanian health system that is expected to provide outpatient care for sick children and are embedded within their communities and thus most likely to be responsive to community feedback [25].

\section{Study design and methodology}

This is a cluster randomized control study with randomization at the health facility level. The study will begin with baseline data collection, followed by implementation of the intervention in the two study arms, followed by endline data collection. Prior to the start of the study we conducted a small qualitative study to inform the quantitative surveys and intervention materials. We conducted four focus group discussions (FGDs) with groups of four-eight parents/guardians of sick children who visited the health facility in the previous seven days. We also conducted four FGDs with groups of four-eight healthcare providers. The FGDs took place at five facilities in the study region, in a district separate from the study districts.

The study includes two rounds of data collection, baseline and end line, at the 75 selected health facilities. We will use two methods of data collection for the parent/guardian surveys. In one district we will conduct phone interviews. Research assistants will approach parents/ guardians after their appointment with the healthcare provider and read a brief recruitment script. If the participants are eligible and agree to participate in the interview 2-7 days later, the research assistants will obtain written consent at the time that their phone number is collected and then follow-up with a phone call. For participants who do not have a phone number to give, we will provide our study phone number and encourage them to 'beep' us from any number they have access to in the following 2-7 days. When we receive phone calls, we will return them to complete the survey. In the remaining three districts, parent/ guardian surveys will be collected as exit surveys. For exit surveys, research assistants will approach caregivers after their visit with the healthcare provider and inform them of the study. If they express interest, the research assistant will invite them to a private space away from the health facility to further inform them of the study.

At both baseline and end line we will also interview healthcare providers at the study facilities. They will be invited to participate in a structured interview that will include questions on socio-demographics, their job satisfaction, and their opinions on quality of care including a free list exercise in conjunction with a discrete choice experiment.

Baseline results will be delivered to intervention facilities after all baseline data collection is complete. Approximately 4-7 weeks after the baseline results have been delivered to the intervention facilities, we will conduct the end line surveys. These will be collected from all study facilities in the same format as outlined above (phone surveys in one district and exit surveys in in three districts). In addition, at end line, to measure the effect of negative patient experiences on health outcomes and patient behavior, we will interview a subset of parents/guardians by phone approximately one to two weeks after their primary visit. We will ask them about the health of the study child and additional questions about recent illness among their children and their careseeking behaviors. However, this study is a proof of concept to detect changes in provider behavior and is not necessarily powered to detect differences in these secondary outcomes. Participants for the follow-up survey will be randomly selected from those who complete the end line parent/guardian survey.

The populations living in the study districts are low-literacy and economically disadvantaged as a whole. We therefore expect that study participants will also be low-literacy and economically disadvantaged. All survey materials are written in language that is understandable to low-literacy populations in the local language (Swahili) and were pilot tested for clarity. The surveys will all be conducted by Swahili-speaking Tanzanian research assistants who have experience working with similar populations. The qualitative data collection conducted prior to the start of the cluster RCT focused on ensuring that survey questions were understandable to this population. All data will be collected using hand-held tablets with the Survey CTO software. Data will be encrypted to protect confidentiality. All research assistants will undergo training in data collection methods, including privacy and confidentiality of participants and their data.

\section{Randomization}

After baseline data collection we will stratify study facilities by district. Within each stratum we will randomize the facilities to one of three arms: private feedback (intervention), social recognition reward (intervention), or no feedback (control) with a 1:1:1 allocation ratio. The principal investigator will conduct randomization using a random number generator in Stata version 14.2. Participants will not be blinded to the allocation.

\section{Intervention}

The study facilities will be randomized into either the control arm or one of two intervention arms: private feedback or public feedback with non-financial reward. The control arm will undergo the same data collection activities as the intervention arm but will not be provided with feedback. If there are changes in patient experience due solely to the act of collecting data, those changes will be captured by this control group. Within both intervention arms, we will give the providers at the study facilities feedback using the baseline data. Feedback will be presented as private feedback reports designed using extensive experience from the U.S. and developing countries [26-28]. The 
feedback reports will provide specific information to make clear which provider behaviors should be changed and will include benchmarks for each indicator. Social comparison will be avoided, as this can lead to reduced performance, especially among low-ability individuals $[16,29]$. The indicators will address specific experiences, be observable by patients, fall into well-identified domains of patient experience, and be within the realm of action by healthcare providers [16,26,28-30].

Facilities randomized to the social recognition reward arm will also have a star-chart displaying their achieved level of patient experience posted at the health facility and village executive officer (village leader's) office. This star design will also be privately given to the healthcare providers at the private feedback facilities so that they have the same information as the public feedback facilities but will not be posted for the community to see. This will allow the providers to understand how to interpret their results, like the providers in treatment arm two, limiting the difference between the treatment arms to the public display of the results.

In addition, facilities in the public feedback arm will receive an encouragement design in the format of recognition from senior officials at the local NGO and/or the Ministry of Health. Providers will be told that the two facilities that score the highest on patient experience indicators and the two facilities that show the most improvement on patient experience indicators at the next round of data collection will receive a letter of recognition from the local NGO and/or the Ministry of Health. Evidence suggests that letters of recognition act as social rewards and lead to improved performance [16].

\section{Participants and selection criteria}

This study involves two groups of participants: parents/guardians of sick children and healthcare providers. Parents/guardians are eligible for inclusion in the study if they are accompanying children under 13 years of age for a sick child outpatient visit at a study facility on a day when we are recruiting patients; are 15 years of age or older; and provides informed consent or assent in the case of parents/guardians aged 15-17. For individuals who are 15-17 years old we will ask if they are either the parent or legal guardian of the child to ensure that they are emancipated minors. For individuals older than 18, all adults who accompanied the child, regardless of their relationship to the child are invited to participate. The relationship to the study child is determined during the survey. For a subset of parents/guardians we will invite them to participate in a follow-up phone survey approximately one to two weeks after their initial facility visit.

All skilled healthcare providers in the study facilities will be invited to participate in a structured interview that assesses their views on healthcare quality as well as their job satisfaction. A healthcare provider is eligible for inclusion in the study if s/he is working in a study facility; is a skilled provider (i.e. cadre is nurse, clinical officer, medical officer, or medical doctor); provides outpatient care for sick children; is 18 years of age or older; and provides informed consent.

\section{Sample size estimation}

Our study sample will include 75 government-managed primary health facilities ( 25 facilities per arm). Our sample size calculations used data from the $\mathrm{MNH}+$ study conducted in the same region in 2016 among 2,002 individuals at 12 health facilities as well as from the Tanzania Demographic and Health Survey 2015-16 [Larson et al. in Press] [24]. We conducted our sample size calculations based on two indicators of patient experience: disrespectful care and provider communication of child's diagnosis, using baseline prevalence and intraclass correlation coefficients (ICC) determined from the prior studies.

For each of the outcomes of interest we calculated the minimum detectable difference given alpha $=0.05$, beta $=0.20,25$ clusters per arm, and 15 individuals per cluster. We calculated the detectable difference assuming a difference in differences design with baseline correlation of 0.8 . All calculations use the clustersampsi command in Stata 14.1. With this sample of 1,125 parents/guardians at baseline and endline, our sample is powered to detect a difference in difference of disrespectful care of 5 percentage points (from 14\% at baseline to $9 \%$ at endline) or a difference in difference in communication of the child's diagnosis of 8 percentage points (from $46 \%$ to $54 \%$ ).

\section{Measures}

The primary outcome of interest is patient experience. To develop measures of patient experience we drew from the literature on respectful maternal care [31], health systems responsiveness [32,33], and patient experience [34]. The final survey questions include previously used measures of disrespectful care in Tanzania and international surveys of patient experience conducted in sub-Saharan Africa by the World Health Survey and the Service Provision Assessment [10,33,35,36]. We also take advantage of publicly available and widely used measures from the U.S. [30]. These indicators are refined and expanded based on our qualitative work. The patient experience includes aspects of patient dignity, autonomy, confidentiality, communication, timely attention, and social support $[32,37]$.

Secondary outcomes are measured through the parent/guardian survey, the parent/guardian follow-up survey, and the healthcare provider surveys. From the parents/guardians we will measure their utilization behavior, rating of technical quality, such as the providers' medical knowledge, satisfaction with care, likelihood of recommending the health facility, rating of overall quality, child's illness resolution, and confidence in the health system. From the healthcare providers we measure attentiveness to patient experience, value of patient experience, perceptions of the quality of care they provide, job satisfaction, stated motivation, and attrition.

While there is no incentive for providers to ask patients to misreport the care received in the control and private feedback arms, there is some concern that providers may ask patients to misreport their care in the reward arm. However, even in this arm the incentive is low, as there is no punishment for poor care and no comparison to other providers. We will ask respondents whether a healthcare provider spoke to them about giving a positive response to the survey.

\section{Identification strategy}

The primary causal identification strategy for the intent to treat analysis will be a difference in difference analysis comparing change in outcomes in the intervention groups to changes in outcomes in the control group through the following model:

$$
Y_{i f}=\mu+\gamma \cdot \operatorname{Pr} \text { ivate }_{i f}+\lambda \cdot T_{i f}+\delta \cdot\left(\operatorname{Pr} \text { ivate }_{i f}+T_{i f}\right)+\eta \cdot \operatorname{Re} \operatorname{ward}_{i f}+v \cdot\left(\operatorname{Re} \operatorname{ward}_{i f} \cdot T_{i f}\right)+\varepsilon_{i f}
$$

Here, Yif is the outcome for individual $i$ in facility $f, \mu$ is the mean outcome among individuals in the control facilities at baseline, $\gamma$ is the mean difference between individuals in the intervention groups and control group at baseline, $\lambda$ is the effect of time ( $\mathrm{T}$ is a post intervention indicator), and $\delta$ and $\eta$ are the estimators of interest: the difference between the change in the private or reward intervention groups and 
the change in the control group, respectively. eif is the individual error clustered at the facility level. We will conduct a sensitivity analysis assessing endline differences only and one imputing missing data. Additional secondary analyses will use discrete choice data from the providers to assess their views on quality of care. Results will be shared with the local and national Ministries of Health in Tanzania and submitted for publication in peer-reviewed journals.Ethical considerations

This study was reviewed and approved by the ethics committees at the Harvard T. H. Chan School of Public Health in the United States and the National Institute for Medical Research in Tanzania prior to the start of data collection. Written informed consent will be obtained by Swahili-speaking, Tanzanian research assistants from all participants prior to participating in the study and will be reminded that they can withdraw at any time. The study will undergo audit at the Harvard T. H. Chan School of Public Health. All spontaneously reported adverse events and other unintended effects of the study will be collected, assessed, and managed by the principal investigator. Adverse events will be reported to the ethics committees. Any updates to the study will go through ethics committee approval and will be included in the PACTR.

\section{Discussion and implications}

The study will assess three causes of poor patient experience: normalization of poor quality, low attention to poor quality, and lack of accountability to the community. Private reports of data on patients' experiences serve to improve providers' awareness of problems by giving providers specific information on areas where patients feel they need improvement. Social recognition by way of public reporting of providers' success in delivering high quality care, incentivize providers to improve and hold them accountable to the communities they serve. This study will provide evidence for which mechanisms affect patient experience and how communities can be involved to improve quality.

If successful, the intervention will provide a method for improving patient experience during outpatient care for sick children. The study will also provide a validated tool for measuring patient experience that could be used in similar contexts, contributing to the body of knowledge on how to measure the quality of care and in particular on the principles to adhere to when measuring quality of care [38].

We expect the study to inform the Ministry of Health, Community Development, Gender, Elderly and Children, Regional and Council Health Management Teams as well as local NGOs on sustainable and efficient means of addressing gaps related to patient experience as contributors to quality care. The current Health Sector Strategic Plan 2015 -June 2020 (HSSP IV) includes plans to promote and strengthen relationships between communities and health facilities, including through empowerment and accountability of health facility governing committees [25]. Tested models for achieving these goals are of great interest to policy makers, both in Tanzania and countries with similar gaps in quality of care.

\section{Declarations}

\section{Ethics approval and consent to participate}

This study was reviewed and approved by the ethics committees at the Harvard T. H. Chan School of Public Health in the United States (IRB17-0931) and the National Institute for Medical Research in Tanzania (NIMR/HQ/R.8a/Vol. IX/2541) prior to the start of data collection.

\section{Consent for publication}

Not applicable

\section{Availability of data and material}

Data sharing not applicable to this article as no datasets were generated or analysed during the current study. The final trial dataset will be accessible by the principal investigator and co-investigators. A minimal, de-identified dataset will be made more widely available within two years of study completion.

\section{Competing interests}

The authors declare that they have no competing interests.

\section{Funding}

This study is funded by The Weiss Family Program Fund for Research in Development Economics. The funder will have no role in collection, management, analysis, and interpretation of data; writing of the report; and the decision to submit the report for publication.

\section{Authors' contributions}

$\mathrm{RM}$ and EE advised on the study design and intervention and provided contextual feedback. RM and EL prepared the first draft. EL conceived the research questions, designed the study, and will conduct data analysis. JC advised on the study design, intervention, and research methods. MZ will supervise data collection. $\mathrm{AB}$ and $\mathrm{SM}$ will supervise the intervention. SC helped prepare the first draft. All authors read and approved the final manuscript.

\section{Acknowledgements}

We would like to thank Dr. Godfrey Mbaruku (Ifakara Health Institute) and Dr. Mwanyika Sando (Africa Academy for Public Health) for their invaluable guidance and support during preparation of the study. We also acknowledge the assistance of Ms. Irene Mashasi in overseeing the qualitative research conducted before the start of the trial and Dr. Adrian Njau for his support on the intervention. Finally, we sincerely thank the RMO of Pwani Region, Dr. Beatrice Byalugaba, as well as the DMOs of the study districts for their support of this protocol. Pan African Clinical Trials Registry: 201710002649121. Protocol version 7, November 8, 2017.

\section{References}

1. Sobel HL, Huntington D, Temmerman M (2016) Quality at the centre of universal health coverage. Health Policy Plan 31: 547-549. [Crossref]

2. Scott KW, Jha AK (2014) Putting quality on the global health agenda. $N$ Engl J Med 371: 3-5. [Crossref]

3. Das J, Hammer J (2014) Quality of Primary Care in Low-Income Countries: Facts and Economics. Annu Rev Econ 6: 525-553

4. Tanzania (2014) Service Delivery Indicators; Health Technical Report. In World Bank Group and African Economic Research Consortium.

5. Larson E, Leslie HH, Kruk ME (2017) The determinants and outcomes of good provider communication: a cross-sectional study in seven African countries. BMJ Open 7: e014888. [Crossref]

6. Abuya T, Warren CE, Miller N, Njuki R, Ndwiga C, et al. (2015) Exploring the prevalence of disrespect and abuse during childbirth in Kenya. PLoS One 10: e0123606. [Crossref]

7. Kruk ME, Kujawski S, Mbaruku G, Ramsey K, Moyo W, et al. (2014) Disrespectful and abusive treatment during facility delivery in Tanzania: a facility and community survey. Health policy and planning 2014. 
8. Sando D, Ratcliffe H, McDonald K, Spiegelman D, Lyatuu G, et al. (2016) The prevalence of disrespect and abuse during facility-based childbirth in urban Tanzania. BMC Pregnancy Childbirth 16: 236. [Crossref]

9. Doyle C, Lennox L, Bell D (2013) A systematic review of evidence on the links between patient experience and clinical safety and effectiveness. BMJ Open 3. [Crossref].

10. Kujawski S, Mbaruku G, Freedman LP, Ramsey K, Moyo W, et al. (2015) Association Between Disrespect and Abuse During Childbirth and Women's Confidence in Health Facilities in Tanzania. Matern Child Health J 19: 2243-2250.

11. Bartlett EE, Grayson M, Barker R, Levine DM, Golden A, et al. (1984) The effects of physician communications skills on patient satisfaction; recall, and adherence. $J$ Chronic Dis 37: 755-764.

12. Greenfield S, Kaplan SH, Ware JE, Yano EM, Frank HJ (1988) Patients' participation in medical care: effects on blood sugar control and quality of life in diabetes. $J$ Gen Intern Med 3: 448-457.

13. Zolnierek KB, Dimatteo MR (2009) Physician communication and patient adherence to treatment: a meta-analysis. Med Care 47: 826-834. [Crossref]

14. Bjorkman M, Svensson J (2009) Power to The People: Evidence from A Randomized Field Experiment of a Community-Based Monitoring Project in Uganda. Power to The People 2009.

15. Bjorkman M, de Walque D, Svensson J (2014) Information is Power: Experimental Evidence on the Long-Run Impact of Community Based Monitoring. Information is Power: 2014, Policy research working paper 7015.

16. Ashraf N, Bandiera O, Lee SS (2014) Awards unbundled: Evidence from a natural field experiment. J Econ Behav Organ 100: 44-63.

17. Ashraf N, Bandiera O, Jack BK (2014) No margin, no mission? A field experiment on incentives for public service delivery. J Public Econ 120: 1-17.

18. Freedman LP, Kruk ME (2014) Disrespect and abuse of women in childbirth: challenging the global quality and accountability agendas. Lancet 384: e42-44. [Crossref].

19. Jamtvedt G, Young JM, Kristoffersen DT, O’Brien MA, Oxman AD (2006) Audit and feedback: effects on professional practice and health care outcomes. Cochrane Database Syst Rev: CD000259. [Crossref]

20. Ketelaar NA, Faber MJ, Flottorp S, Rygh LH, Deane KH, et al. (2011) Public release of performance data in changing the behaviour of healthcare consumers, professionals or organisations. Cochrane Database Syst Rev CD004538. [Crossref]

21. McNamara P (2006) Provider-specific report cards: a tool for health sector accountability in developing countries. Health policy plan 21: 101-109.

22. Molyneux S, Atela M, Angwenyi V, Goodman C (2012) Community accountability a peripheral health facilities: a review of the empirical literature and development of a conceptual framework. Health Policy Plan 27: 541-554. [Crossref]

23. Pwani Region Socio-economic profile (2013) In Dar es Salaam, Tanzania: Ministry of Finance, National Bureau of Statistics, and Pwani Regional Secretariat.
24. Tanzania Demographic and Health Survey and Malaria Indicator Survey (2016) Final Report. In. Dar es Salaam, Tanzania and Rockville, Maryland, USA: Ministry of Health, Community Development, Gender, Elderly and Children (MoHCDGEC) Tanzania Mainland, Ministry of Health (MoH) Zanzibar, National Bureau of Statistics (NBS), Office of the Chief Government Statistician (OCGS), and ICF.

25. Welfare URoTMoHaS (2015) Health sector strategic plan July 2015-June 2020 (HSSP IV); Reaching all household with quality health care.

26. Hibbard J, Sofaer S (2010) Best practices in public reporting No. 1: How to effectively present health care performance data to consumers. In Agency for Healthcare Research and Quality.

27. Schlesinger M, Grob R, Shaller D (2015) Using Patient-Reported Information to Improve Clinical Practice. Health services research 50: 2116-2154. [Crossref]

28. Shaller D, Kanouse D (2012) Private "Performance Feedback" reporting for physicians; guidance for community quality collaboratives. Agency for Healthcare Research and Quality.

29. Brock J, Lange A, Leonard KL (2014) Generosity and prosocial behavior in health care provision: evidence from teh laboratory and field. Working paper.

30. Anhang R, Elliott MN, Zaslavsky AM, Hays RD, Lehrman WG, et al. (2014) Examining the Role of Patient Experience Surveys in Measuring Health Care Quality. Med Care Res Rev 71: 522-554. [Crossref]

31. Brickson KBE, Deller B, Warthin C, Lunardi N (2013) Respectful maternity care measurement workshop report. Maternal and Child Health Integrated Program (MCHIP).

32. Valentine N, Darby C, Bonsel GJ (2008) Which aspects of non-clinical quality of care are most important? Results from WHO's general population surveys of "health systems responsiveness" in 41 countries. Soc Sci Med 66: 1939-1950. [Crossref]

33. Valentine N, Verdes-Tennant E, Bonsel G (2015) Health systems' responsiveness and reporting behaviour: Multilevel analysis of the influence of individual-level factors in 64 countries. Soc Sci Med 138: 152-160. [Crossref].

34. Toomey SL, Zaslavsky AM, Elliott MN, Gallagher PM, Fowler FJ, et al. (2015) The Development of a Pediatric Inpatient Experience of Care Measure: Child HCAHPS(R) Pediatrics 136: 360-369. [Crossref]

35. Tanzania Service Provision Assessment Survey (2015) Preliminary Report. Ministry of Health and Social Welfare: Dar es Salaam, Ministry of Health: Zanzibar, National Bureau of Statistics: Dar es Salaam, Office of Chief Government Statistician: Zanzibar, ICF International: Rockville, Maryland USA.

36. Larson E, Hermosilla S, Kimweri A, Mbaruku GM, Kruk ME (2014) Determinant of perceived quality of obstetric care in rural Tanzania: a cross-sectional study. BMC Health Serv Res 14: 483. [Crossref]

37. World Health Organization (2000) The world health report. Health systems: improving performance. Geneva.

38. Hanefeld J, Powell-Jackson T, Balabanova D (2017) Understanding and measuring quality of care: dealing with complexity. Bull World Health Organ 95: 368-374. [Crossref]

Copyright: (C2018 Mbatia R. This is an open-access article distributed under the terms of the Creative Commons Attribution License, which permits unrestricted use, distribution, and reproduction in any medium, provided the original author and source are credited. 